\title{
Update on the diagnosis and management of early rheumatoid arthritis
}

\author{
Author: Peter C Taylor ${ }^{\mathrm{A}}$
}

The clinical spectrum of rheumatoid arthritis (RA) presentation is heterogeneous, with wide variation in age of onset, degree of joint involvement and severity. Delayed rheumatological referral is associated with less favourable long-term outcomes. Multidisciplinary teamwork is key to the success of a holistic approach to patient care and addressing issues that are important to the person. Pharmacological treatment of early RA aims to optimally suppress the inflammatory component of the disease with an ideal treatment target of remission. The overarching aim of management of early RA is to achieve a quality of life that permits the individual to pursue normal work, domestic and social activity.

\section{Introduction}

Rheumatoid arthritis (RA) is a systemic inflammatory syndrome with autoimmune features with a predominant expression in peripheral synovial joints. It is the commonest form of inflammatory polyarthritis with a population prevalence of between $0.5 \%$ to $1 \%{ }^{1}$. Once established and, if persistently active, RA generally becomes straightforward to recognise and is characterised by a deforming symmetrical polyarthritis, although the extent and severity differ widely. However, the variability of the presentation in the early stages of the illness is such that diagnosis, or classification, can be very difficult. Given the high prevalence and unequivocal long-term benefits of early recognition and therapeutic intervention, whether the diagnosis is beyond doubt or uncertain, it is important that physicians are aware of this common condition and refer suspected cases promptly for specialist evaluation and care.

\section{Clinical presentation}

The clinical presentation of RA is heterogeneous, as is the subsequent disease course. It may begin at any age with a wide spectrum of onset, although patients most commonly present from the sixth decade onwards with a peak incidence between 70 and 80 years of age. Women are affected about twice as commonly as men in the UK. ${ }^{2}$

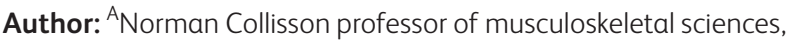
University of Oxford, Oxford, UK
RA often begins insidiously, with slow development of signs and symptoms, which may vary in intensity, over many weeks. These may include stiffness in one or more joints, often most marked in the morning, accompanied by discomfort on movement and joint tenderness. Over time, the pattern may become more persistent and tends to evolve into symmetrical, polyarticular involvement (comprising five or more joints). The joint distribution frequently involves the metacarpophalangeal (MCP) and proximal interphalangeal joints of the hands, typically with sparing of the distal interphalangeal joints, the wrists, and metatarsophalangeal (MTP) joints of the feet. Patients may report that they feel as if they are walking on pebbles and that they have difficulty in making a full fist in the morning. In many individuals, large joints are also affected including the shoulders, elbows, knees and ankles. The cervical vertebrae may also become involved while the spine is otherwise unaffected.

\section{Key points}

Rheumatoid arthritis (RA) is the commonest form of inflammatory polyarthritis with a population prevalence up to $1 \%$.

The 'diagnosis' of RA is made based on clinical judgement, but contemporary classification criteria are designed to promote prompt treatment intervention.

Rapid referral to a secondary care specialist is indicated when features of persistent inflammatory arthritis are suspected.

Early initiation of conventional synthetic disease modifying anti-rheumatic drug treatment greatly reduces the risk of future joint damage and disability.

A multidisciplinary approach to RA management is invaluable to address symptoms of concern to patients.

KEYWORDS: Rheumatoid arthritis, disease-modifying anti-rheumatic drugs, clinical assessment, methotrexate, treat-to-target

DOI: $10.7861 /$ clinmed.2020-0727 
Other less common articular patterns of RA presentation may also occur. These include an acute, explosive, polyarticular onset which may arise overnight or over the course of a few days. Another is a so-called 'palindromic' presentation, in which transient symptoms arise in few joints over days to weeks before completely resolving, only to recur later in the same or other joints and tending to increase over time. In more elderly patients, RA may present as steroid-responsive polymyalgia rheumatica and the more typical peripheral, symmetrical arthritis of established RA may only become apparent once the steroid dose is weaned down.

Prolonged morning joint stiffness is highly suggestive of inflammatory arthritis as distinct from the transient stiffness of osteoarthritis, which lasts a few minutes only. Morning joint stiffness persisting for over an hour, and often lasting several hours, is especially characteristic of RA and tends to worsen with disease activity. Prolonged joint stiffness may also occur after daytime inactivity.

Joint symptoms may be preceded and accompanied by a variety of nonspecific symptoms including debilitating fatigue, malaise and depression which tend to reflect the level of systemic inflammation. Such symptoms may vary greatly in intensity from one day to another and even throughout the day. Low grade fever may be another feature of systemic inflammation.

Early in the course of RA there is a high correlation between disability and inflammation and little relationship to radiographic damage. ${ }^{3}$ However, as the condition becomes more established, if joint destruction accrues, structural damage becomes a major determinant of functional disability. ${ }^{4}$

\section{Clinical assessment}

Symptoms associated with RA should be assessed by means of a descriptive history and attempting to quantify their severity. Similarly, signs of RA should be documented following careful examination. Synovial thickening is detected on palpation as a spongy or boggy feel, and tenderness is elicited by squeezing an affected joint. Discomfort elicited by squeezing of the MTP joints of the feet is a sensitive indication of possible RA. Joint effusions can be demonstrated by fluctuation. As RA progresses, deformities may advance and surfaces of subluxed bones may falsely give the impression of joint swelling. This is particularly evident at the heads of the MCP joints, the ulnar styloid and the distal radius at the wrist. RA disease activity can be evaluated by means of one of several validated formulae for composite scores, components of which usually include the number of swollen and tender joints out of a defined set assessed by the rheumatologist as well as a physician assessment of global status. Other components included in various composite measures include acute phase reactant levels and a patient assessment of global status.

\section{Investigations}

There are no laboratory tests that are pathognomonic for RA. Anti-cyclic citrullinated protein antibody (ACPA) and/or immunoglobin $\mathrm{M}$ rheumatoid factor can be detected in blood in about three-quarters of patients, sometimes preceding symptom onset by years, and are relatively specific for this condition. ${ }^{5}$ However, neither of these tests are sufficiently specific to establish the classification of RA, and prognosis varies widely within seropositive and seronegative patient populations. Other laboratory measures include acute phase inflammatory markers such as C-reactive protein levels. Plain X-rays of the hands and feet at first presentation are useful to assess structural damage and whether there is evidence of erosive disease at baseline. Ultrasound has high sensitivity for early detection of erosions in the hands and feet and is also widely used to evaluate of soft tissue involvement. Ultrasound can supplement clinical examination for the detection of subclinical synovitis. It is also valuable in assessment of tenosynovitis and involvement of digit flexors in early arthritis is predictive of persistence. ${ }^{6}$ Ultrasound is readily acceptable to patients and can be helpful in the context of shared decision making by visibly demonstrating the presence or absence of inflammation to the patient.

\section{Diagnosis and classification}

The 'diagnosis' of RA is made based on clinical judgement. But because of the heterogeneity of presentation, and the compelling evidence that best long-term outcomes can be achieved by effective, early intervention, updated classification criteria were published in 2010 by the American College of Rheumatology (ACR) and European League Against Rheumatism (EULAR) with a view to encouraging timely introduction of therapy. ${ }^{7}$ These criteria apply a weighted score to each of four domains comprising joint distribution, serology, presence of an acute phase response and symptom duration. A score of 6 or more out of a possible maximum of 10 constitutes a classification of 'definite' RA (Table 1).

\section{Differential diagnosis}

The differential diagnosis is potentially broad including, but not limited to, psoriatic arthritis, haemochromatosis, sarcoidosis and

\section{Table 1. The 2010 American College of}

Rheumatology (ACR) and European League Against Rheumatism (EULAR) classification criteria for rheumatoid arthritis ${ }^{7}$

\section{Joints distribution (0-5)}

$>1$ large joint

$>$ 2-10 large joints

$1-3$ small joints (large joints not counted)

4-10 small joints (large joints not counted)

$>10$ joints (at least 1 small joint)

\section{Serology (0-3)}

$>$ Negative RF AND negative ACPA

$>$ Low positive RF OR low positive ACPA

$>$ High positive RF OR high positive ACPA

\section{Symptom duration (0-1)}

$><6$ weeks

$>\geq 6$ weeks

0

Acute phase reactants (0-1)

> Normal CRP AND normal ESR > Abnormal CRP OR abnormal ESR

Total score $\geq 6$ = classification of 'definite' RA. The score can be calculated prospectively over time (cumulatively) or retrospectively if data have been recorded in the past. ACPA = anti-cyclic citrullinated protein antibody; CRP = $\mathrm{C}$-reactive protein; $\mathrm{ESR}=$ erythrocyte sedimentation rate; $\mathrm{RF}=$ rheumatoid factor. 
other forms of polyarthritis associated with various connective tissue diseases including systemic lupus erythematosus and primary Sjogren's syndrome. Polyarthritis may also be the presenting feature of a variety of infectious agents including rubella, parvovirus B-19, Lyme disease or reactive arthritis associated with infections of the genito-urinary or gastrointestinal tract. Another differential diagnosis that may cause diagnostic difficulty in elderly patients includes polyarticular chronic pyrophosphate arthropathy.

\section{Pharmacological management}

The primary goal of treating patients with RA is to maximise longterm health-related quality of life through control of symptoms, prevention of structural damage, normalisation of function, and participation in social and work-related activities. Referral to a rheumatologist for very early initiation of conventional synthetic disease-modifying anti-rheumatic drug (csDMARD) treatment, before the onset of erosions, greatly reduces the risk of future joint damage and disability. ${ }^{8,9}$ The most prescribed csDMARDs include methotrexate, leflunomide, sulfasalazine and hydroxychloroquine. EULAR recommends treatment with CSDMARDs upon initiation of treatment for RA. ${ }^{10}$ Short-term glucocorticoid use should be considered when initiating or changing csDMARDs but should be tapered as rapidly as clinically feasible. ${ }^{10}$ Methotrexate is currently the most used first-line therapy for RA in the world. ${ }^{11}$ Methotrexate monotherapy is recommended as an initial pharmacological strategy but it can also be used as an 'anchor drug' in combination with other csDMARDs in the management of early RA, and with target biologic or small molecule therapy in established disease. Based on its efficacy, safety, large dose-titratable range, options for either an oral or parenteral route of administration, and cost-effectiveness, methotrexate holds a unique place in the management of RA. ${ }^{12}$ Recommended practice is to treat to a target of remission or low disease activity, as assessed by composite scores of disease activity, and to titrate therapy according to response. ${ }^{13}$ However, many patients with established RA do not achieve treatment targets with methotrexate monotherapy which may also have issues with gastrointestinal tolerability. ${ }^{14}$ If oral methotrexate is ineffective or not tolerated, there is an option to switch to subcutaneously administered drug. Whereas the bioavailability of oral methotrexate reaches a plateau at doses of $15 \mathrm{mg} /$ week, the higher and less variable bioavailability of subcutaneous methotrexate, combined with its linear dose-dependency, make it an attractive option for switching if titration of oral methotrexate fails to produce an adequate response. ${ }^{15}$ If the treatment target is not achieved with the first csDMARD strategy, when poor prognostic factors are present, addition of a targeted therapy should be considered. ${ }^{10}$

\section{Non-pharmacological management}

Pharmacological intervention represents only one aspect of the management plan for RA. A multidisciplinary approach is invaluable to address other important aspects of care including patient education, including evaluation of coping with activities of daily living with appropriate access to aids and adaptations, as well as podiatry support, and psychological and employment counselling if required.

\section{Conclusion}

RA is the commonest form of inflammatory polyarthritis, but it is not always straightforward to recognise at onset because of the variability of presentation. However, early therapeutic intervention has unequivocal long-term benefits in prevention of disability and preservation of quality of life. Features of an inflammatory arthritis suggesting that rapid referral to a secondary care specialist is indicated include the presence of more than three swollen joints; a positive squeeze test of MTP or MCP joints; MCP or wrist joint swelling; and morning joint stiffness persisting more than 30 minutes. As a consequence of early intervention, the advent of highly efficacious targeted therapies and a treat-to-target approach to management, the outlook for people presenting with RA has improved dramatically within one generation. ${ }^{16}$

\section{Acknowledgements}

Thanks to the National Institute of Health Research (NIHR) for their funding of the NIHR Biomedical Research Centre in musculoskeletal disease at Oxford University Hospitals NHS Foundation Trust and the University of Oxford.

\section{References}

1 Gabriel SE. The epidemiology of rheumatoid arthritis. Rheumatic Disease Clinics of North America 2001;27:269-81.

2 Abhishek A, Doherty M, Kuo CF et al. Rheumatoid arthritis is getting less frequent-results of a nationwide population-based cohort study. Rheumatology (Oxford) 2017;56:736-44.

3 Kirwan JR. Links between radiological change, disability, and pathology in rheumatoid arthritis. J Rheumatol 2001;28:881-6.

4 Taylor PC, Law ST. When the first visit to the rheumatologist is established rheumatoid arthritis. Best Pract Res Clin Rheumatol 2019;33:101479.

5 Taylor PC. Biologic markers in the diagnosis and assessment of rheumatoid arthritis. UpToDate 2019. www.uptodate.com/ contents/biologic-markers-in-the-diagnosis-and-assessment-ofrheumatoid-arthritis

6 Sahbudin I, Pickup L, Nightingale P et al. The role of ultrasounddefined tenosynovitis and synovitis in the prediction of rheumatoid arthritis development. Rheumatology (Oxford) 2018;57;1243-52.

7 Aletaha D, Neogi T, Silman AJ et al. Rheumatoid arthritis classification criteria: an American College of Rheumatology/ European League Against Rheumatism collaborative initiative. Arthritis Rheum 2010;62:2569-81.

8 Smolen JS, Aletaha D, Koeller $M$ et al. New therapies for treatment of rheumatoid arthritis. Lancet 2007:370:1861-74.

9 Combe B, Landewe R, Daien CI et al. 2016 update of the EULAR recommendations for the management of early arthritis. Ann Rheum Dis 2017;76:948-59.

10 Smolen JS, Landewe R, Bijlsma ] et al. EULAR recommendations for the management of rheumatoid arthritis with synthetic and biological disease-modifying antirheumatic drugs: 2016 update. Ann Rheum Dis 2017;76:960-77.

11 Weinblatt ME. Methotrexate in rheumatoid arthritis: A quarter century of development. Trans Am Clin Climatol Assoc 2013;124: $16-25$.

12 Taylor PC, Balsa Criado A, Mongey A-B et al. How to get the most from methotrexate (MTX) treatment for your rheumatoid arthritis patient? - MTX in the treat-to-target strategy. J Clin Med 2019;8:515-45. 
13 Smolen JS, Breedveld FC, Burmester GR et al. Treating rheumatoid arthritis to target: 2014 update of the recommendations of an international task force. Ann Rheum Dis 2016:75:3-15.

14 Braun ], Kästner P, Flaxenberg P et al. Comparison of the clinical efficacy and safety of subcutaneous versus oral administration of methotrexate in patients with active rheumatoid arthritis. Arthritis Rheum 2008;58:73-81.

15 Schiff MH, Jaffe JS, Freundlich B. Head-to-head, randomised, crossover study of oral versus subcutaneous methotrexate in patients with rheumatoid arthritis: Drug-exposure limitations of oral methotrexate at doses $\geq 15 \mathrm{mg}$ may be overcome with subcutaneous administration. Ann Rheum Dis 2014;73:1549-51.

16 Taylor PC, Pope J. Treating to target or treating the patient in rheumatoid arthritis? Lancet Rheumatology 2019;1:e8-10.

Address for correspondence: Prof Peter C Taylor, Botnar Research Centre, Nuffield Department of Orthopaedics, Rheumatology and Musculoskeletal Sciences, University of Oxford, Windmill Road, Headington, Oxford OX3 7LD, UK. Email: peter.taylor@kennedy.ox.ac.uk

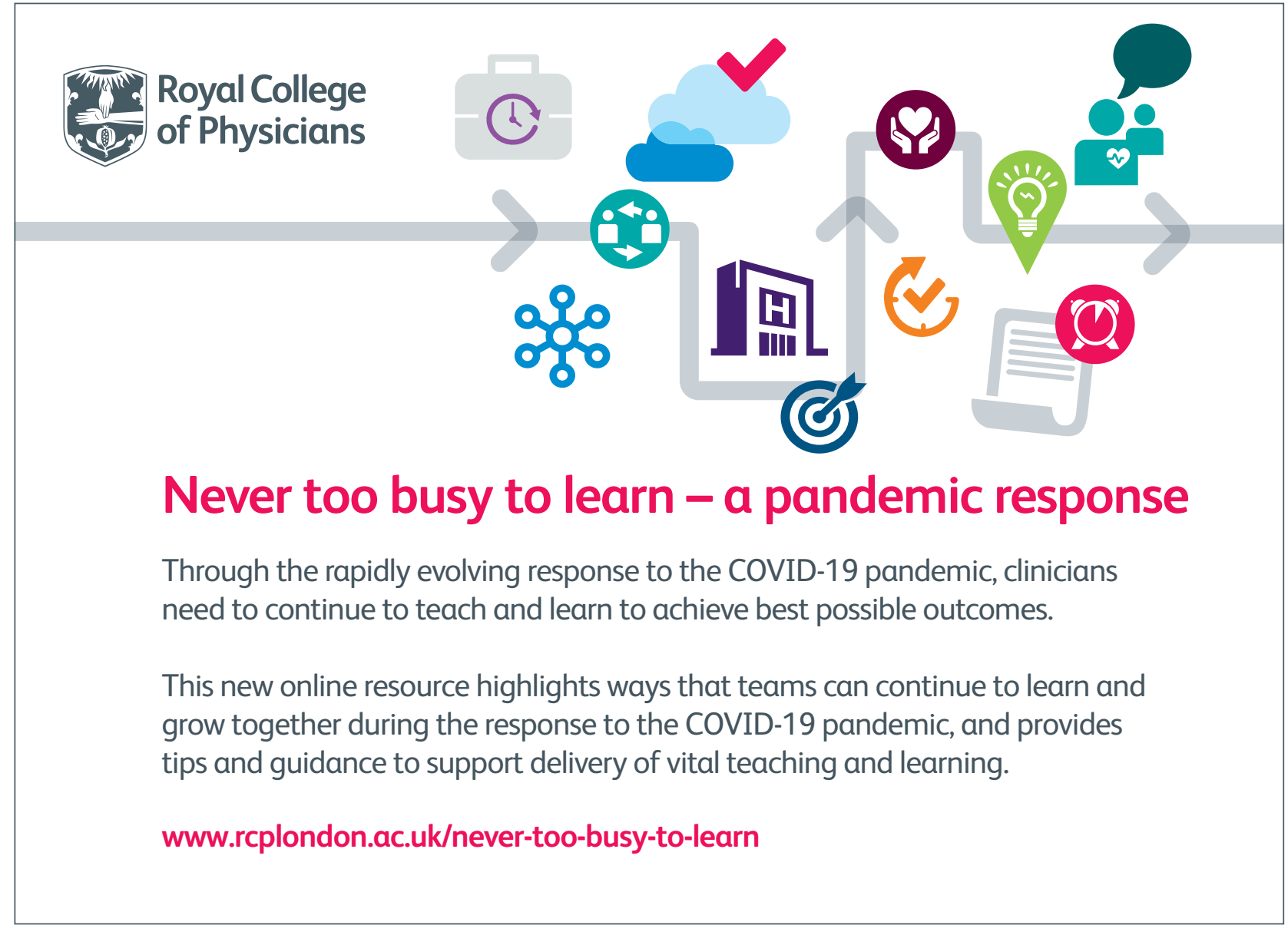

